\title{
Stereo-PIV study of flow inside an eye under cataract surgery
}

Jun Sakakibara*, Masaki Yamashita*, Tatsuya Kobayashi*, Yuichi Kaji**, Tetsuro Oshika**

* Department of Engineering Mechanics and Energy, University of Tsukuba, Tennodai 1-1-1, Tsukuba, Ibaraki, 305-8573, Japan

** Department of Ophthalmology, Institute of Clinical Medicine, University of Tsukuba, Tennodai 1-1-1, Tsukuba, Ibaraki, 305-8573, Japan

Corresponding author: Jun Sakakibara

Phone: 81-298-53-6189

Fax: 81-298-53-5208

E-mail: sakakiba@kz.tsukuba.ac.jp 


\section{Abstract}

We measured velocity distributions in the anterior chamber of porcine eyes under simulated cataract surgery using stereoscopic particle image velocimetry (stereo-PIV). The surface of the cornea was detected based on the images of laser-induced fluorescent light emitted from fluorescent dye solution introduced in a posterior chamber. A coaxial phacoemulsification procedure was simulated with standard size (standard coaxial phacoemulsification) and smaller (micro coaxial phacoemulsification) surgical instruments. In both cases, an asymmetric flow rate of irrigation was observed, although both irrigation ports had the same dimensions prior to insertion into the eye. In cases where the tip of the handpiece was placed farther away from the top of the cornea, i.e., closer to the crystalline lens, direct impingement of irrigation flow onto the cornea surface was avoided and the flow turned back towards the handpiece along the surface of the corneal endothelium. Viscous shear stress on the corneal endothelium was computed based on the measured mean velocity distribution. The maximum shear stress for most cases exceeded 0.1 $\mathrm{Pa}$, which is comparable to the shear stress that caused detachment of the corneal endothelial cells reported by Kaji et al. [Cornea 2005; 24:S55-S58]. When direct impingement of the irrigation flow was avoided, the shear stress was reduced considerably. 


\section{Introduction}

A cataract is an opacity that develops in the crystalline lens of the eye. The most effective treatment for a cataract is phacoemulsification cataract surgery, a procedure in which the crystalline lens is broken and then removed by an ultrasonic irrigation and aspiration hand piece, and then replaced by an artificial lens. A currently popular version of this procedure is 'coaxial' phacoemulsification, in which the hand piece consists of a combination of a stainless pipe, called the 'tip,' and a surrounding silicone tube, called the 'sleeve,' as shown schematically in Fig. 1. The tip vibrates at an ultrasonic frequency to break the crystalline lens and send the emulsified lens material to a tube pump installed in the surgical equipment to be aspirated through the aspiration port at the end of the pipe. The sleeve is coaxially arranged over the tip, and a gap between the tip and the sleeve is connected to an overhead bottle to create irrigation flow through the irrigation port of the sleeve. The irrigation keeps pressure in the anterior chamber while the broken crystalline lens and fluid are being aspirated.

Although the irrigation and aspiration flow are necessary for the removal of the crystalline lens, they create circulation in the chamber, while the ultrasound wave induces acoustic streaming from the phacoemulsification tip, and this flow can damage the corneal endothelial cells [1-7]. Even hydrodynamic forces acting on the endothelial cells without sonification or rubbing of the nuclear materials is deleterious to the endothelium [7]. The tangential component of the hydrodynamic forces, i.e., shear stress, affects the endothelial cells such as vascular endothelial cells [8], hybridomas [9] and even cornea endothelial cells [10].

Although accurate estimation of the shear stress acting on the cornea endothelium under phacoemulsification is important for clarifying the physical mechanism of the endothelial cell damage, no quantitative information has been given in the literature to date. In the present study our goal was to study the time-averaged velocity distribution in the anterior chamber using stereoscopic particle image velocimetry (stereo-PIV) [11] to visualize the flow pattern during simulated coaxial phacoemulsification. Based on the measured velocity distribution, we estimated the hydrodynamic shear stress on the corneal endothelium as a way to evaluate the mechanical effect on the cell structures. The position of the surface of the corneal endothelium, i.e., the wall position, which is crucial for the estimation of the shear stress, was determined based on the fluorescent images of fluorescence solution in the anterior chamber. 


\section{Method}

\subsection{Experimental apparatus and instrumentation}

Figure 2 shows an overview of the experiment. An eye extracted from a pig was fixed on a Styrofoam block with its cornea directed upward. A surgical handpiece connected to a control unit (Infiniti, Alcon, Inc.) was held horizontally with a biaxial traversing stage, and its tip was inserted in the eyeball through an incision made on the edge of the cornea. The aspiration rate and height of the irrigation bottle of the control unit were set and fixed through each individual run of the experiment. A Nd-YAG laser beam (30 mJ/pulse, 15 Hz, Solo PIV, New Wave Research, Inc.) passed through a combination of two cylindrical lenses, forming a light sheet with a thickness of approximately $1 \mathrm{~mm}$, and illuminated a vertical plane of the posterior chamber of the eye through the cornea. Two CCD cameras (1392x1040 pixels, $30 \mathrm{fps,} \mathrm{TM-1325CL,} \mathrm{JAI/Pulnix,} \mathrm{Inc.),} \mathrm{each}$ with a Nikon lens (Nikon Micro $105 \mathrm{~mm}, \mathrm{~F} 2.8$ ), were positioned at a level higher than the eye and inclined toward it. The angle between the optical axes of the two CCD cameras was approximately 70 degrees, and the Scheimpflug condition was satisfied. To reduce the astigmatism aberration due to the inclined air-water interface, a prism was fixed on the water surface. The Cartesian coordinate system was used, as shown in Figure 3. The origin was set at the head of the tip.

Stereoscopic PIV, which resolves the instantaneous out-of-plane velocity component in addition to the in-plane component, was used to measure the three-component velocity in the laser light sheet. Details of the algorithm used in this study were presented previously [12]. To calibrate the physical position in the acquired images, we placed a calibration plate with $0.2-\mathrm{mm}$-diameter holes at $0.5-\mathrm{mm}$ intervals in its surface in a thin volume of the light sheet. Since it was not possible to place the calibration plate directly in the posterior chamber of the eye, the plate was placed at the position of the light sheet plane just outside the eye. It is possible that this calibration procedure could have caused an error due to the refraction of the laser light and the scattering light emanating from the particle; however, based on our optical analysis, we estimated the maximal potential error to be no more than $0.05 \mathrm{~mm}$. As this is negligibly small, we believe the refraction did not affect our conclusion. The third-order polynomial warping functions were created by the calibration procedure and then used in the stereo-PIV software created in-house.

The region captured by cameras was parallelogram-shaped with each side $12 \mathrm{~mm}$ in length, as shown in Fig. 6. The interrogation spot size in the raw image was 30x30 pixels. Spatial resolution of the velocity measurement was approximately $0.26 \mathrm{~mm}$ and $0.35 \mathrm{~mm}$ in the $x$ - and $y$-directions, respectively, which is identical to the interrogation spot size, and $1 \mathrm{~mm}$ in the $z$-direction, which is identical to the light sheet thickness. Temporal separation of the PIV image pairs was $70 \mu \mathrm{s}$ in most cases. 


\subsection{Tracer particles}

Fluorescent liposomes were used as the tracer particles in this study. Liposomes are suitable tracers for visualizing the biological flow bounded on the cellular tissue, since they are less adhesive to the cornea surface than are regular plastic or metal particles. The liposomes were stained by fluorescent dye to emit fluorescent light with a wavelength longer than that of laser light, so we could distinguish the light coming from the tracer particles and the light scattered from surfaces such as the cornea or iris. The fluorescent liposomes were prepared as follows.

Fluorescent dye Rhodamine 6G was dissolved in egg yolk phospholipids (Coatsome NC-50, NOF Corp., Japan). The mixture was then dissolved in methanol with a resulting concentration of 15 $\mathrm{mg} / \mathrm{ml}$, and 1.5-ml aliquots were poured into Petri dishes (Corning Inc., NY). The methanol on the dishes was then dried in vacuum desiccators for $24 \mathrm{~h}$. After the addition of $5 \mathrm{~mL}$ of distilled water to each dish, the liposomes with phospholipids bilayer membranes were formed. To avoid diffusion of the dye out of the liposomes, the liposomes were centrifuged once to eliminate the supernatant solution, and then distilled water was added and they were centrifuged again. After eliminating the supernatant, each fluorescent liposome was ready for the experiment. The diameter of the liposomes ranged from 5 to $30 \mu \mathrm{m}$.

\subsection{Eyeballs}

Porcine eyes were purchased from a slaughterhouse. The eyes were soaked in saline water and kept at approximately 4 degrees $\mathrm{C}$ until use. The mean size of the anterior chamber of 26 porcine eyes in the $z$-direction (along the major axis) was approximately $15.7 \mathrm{~mm}$ with a standard deviation of $0.65 \mathrm{~mm}$ and in the $x$-direction (along the minor axis) was approximately $12.85 \mathrm{~mm}$ with a standard deviation of $0.48 \mathrm{~mm}$.

\subsection{Handpiece}

The surgical methods we simulated in this study were the standard coaxial surgery and micro coaxial surgery. The latter utilizes a smaller diameter of tip and sleeve with a smaller incision than those of the standard coaxial surgery. The tip and sleeve used in this study are shown in Fig. 4. For the standard coaxial surgery, the tip and the sleeve were the " $0.9 \mathrm{~mm}$ micro ABS tip $30^{\circ}$, Alcon, Inc." and the "0.9 mm micro sleeve, Alcon, Inc.," respectively. The combined tip and sleeve device was inserted through a 3-mm incision of cornea. For the micro coaxial surgery, the tip and the sleeve were the " $0.9 \mathrm{~mm}$ micro flared ABS tip $30^{\circ}$, Alcon, Inc." and the "0.9 mm ultra sleeve, Alcon, Inc," respectively. The incision for this case was $2.2 \mathrm{~mm}$.

We performed the standard coaxial surgery on three eyeballs and the micro coaxial surgery on three eyeballs. For each case the head of the tip was positioned at different locations in the anterior chamber, as summarized in Table 1. The parameters used in this table are explained in Fig. 5. For all cases, ultrasonic vibration of the tip, which is normally used to break the lens, was deactivated. The irrigation bottle was $90 \mathrm{~cm}$ above the handpiece, and the aspiration rate was $30 \mathrm{~mL} / \mathrm{min}$. These settings are frequently chosen in actual cataract surgery. The handpiece was inserted 
horizontally into the posterior chamber through an incision made on the edge of the cornea. Although the pressure inside the anterior chamber was not measured, the pressure was lower than the hydrostatic pressure derived from the bottle height, since the significant amount of energy loss in the hand piece and the gap between the sleeve and the tip reduced the pressure.

\subsection{Estimation of shear stress}

Shear stress acting on the corneal interior surface was computed based on the velocity gradient on the surface and fluid viscosity. To estimate the velocity gradient on the surface, the precise location of the surface must be known. We determined the location of the surface based on laserinduced fluorescence. Organic fluorescent dye Rhodamine $6 \mathrm{G}$ was dissolved in the saline water used as irrigation fluid at a concentration of $20 \mathrm{mg} / \mathrm{L}$, and the mixture was supplied through the handpiece into the posterior chamber. After the posterior chamber was filled with fluorescent dye and we made sure the dye concentration was uniform in the chamber, fluorescence images were captured by the cameras. A typical fluorescence image is shown in Fig. 6. The cornea surface can be observed at the upper boundary of the bright and dark region, although it is blurred and hard to determine its exact location. The exact location of the surface was determined using the following method.

Schematic representation of the projection of the fluorescent light onto the camera is provided in Fig. 7. A mid-plane of the laser light sheet was assumed to be extended into the $x-y$ plane through the $y$-axis $(z=0)$. Fluorescent light emitted from a molecule is projected onto the CCD sensor along a line designated by the axis $\xi$, which intersects with the $y$-axis at the coordinate $y$. The fluorescence intensity profile, $I(\xi)$, along the axis $\xi$ has a shape similar to, but wider than and a part of, the profile of laser light intensity. Assuming that the laser light intensity profile is Gaussian, the fluorescence profile is expressed as follows:

$$
I(\xi)=A e^{-\xi^{2} / \sigma^{2}}
$$

Here, $A$ is a constant and $\sigma$ is the $1 / e$-width of the profile. Denoting $\xi_{\mathrm{I}}$ as a distance measured along the $\xi$-axis from the $y$-axis to the interior surface of cornea, $\xi_{\mathrm{I}}$ is determined by

$$
\xi_{\mathrm{I}}=\frac{y_{0}-y}{\sin \beta-\tan \alpha \cos \beta},
$$

where the curvature of the surface was ignored, $y_{0}$ is the location of the cornea surface along the $y$ axis, and $\alpha$ and $\beta$ are the angle of the $\xi$-axis and cornea surface with respect to the $z$-axis, respectively.

The total amount of fluorescent light intensity projected on the CCD sensor along the $\xi$-axis is

$$
E(y)=\int_{-\infty}^{\xi_{1}} I(\xi) d \xi
$$

Combining eq. (1)-(3), the second-order derivative of $E$ becomes 


$$
\frac{d^{2} E}{d y^{2}}=-\frac{2 A\left(y_{0}-y\right)}{\sigma^{2}(\sin \beta-\tan \alpha \cos \beta)^{3}} e^{-\frac{\left(y_{0}-y\right)^{2}}{(\sin \beta-\tan \alpha \cos \beta)^{2} \sigma^{2}}}
$$

This yields that the inflection point of the fluorescence intensity profile in the image,

i.e. $d^{2} E / d y^{2}=0$, represents the location of the cornea surface where $y=y_{0}$. Thus the secondorder derivative of the profile was computed and indicated where the value became zero to determine the location of the cornea surface. We used this method to determine the boundary of the crystalline lens and posterior chamber. An example of the boundary computed by the above method is shown by a solid line in Fig. 6. Note that the error in the cornea location associated with linear approximation of the curved surface was estimated to be $0.01 \mathrm{~mm}$ when the radius of the curved surface was $10 \mathrm{~mm}$, and the 1/e thickness of the light sheet was $1.0 \mathrm{~mm}$.

To justify the use of this method, we prepared a rectangular aluminum container with a glued glass plate on the top, filled with fluorescent solution. Submerging this container into the location where the eye ball was fixed, a fluorescence image was captured and the location of the interior surface below the glass plate was estimated using the method just described. As shown in Fig. 8 the estimated location of the glass surface was in good agreement with its actual location. The maximum error found in this figure is approximately $50 \mu \mathrm{m}$.

Local viscous shear stress $\tau_{w}$ acting on a surface element at a given point $\boldsymbol{x}$ on the cornea is represented by

$$
\tau_{w}(\mathbf{x})=\left.\mu \frac{d u_{t}(\mathbf{x}, h)}{d h}\right|_{h=0} \cong \mu \frac{u_{t}(\mathbf{x}, \Delta h)}{\Delta h},
$$

where $\mu$ is the fluid viscosity, $u_{\mathrm{t}}$ is a tangential component of the velocity with respect to the corneal surface, and $h$ and $\Delta h$ represent the distance from the given point $x$ along the line normal to the surface. The finite distance $\Delta h$ was chosen to be $0.75 \mathrm{~mm}$ to avoid the overlap of the PIV interrogation volume and the surface. Since velocity data were discrete in space, $u_{t}(\mathbf{x}, \Delta h)$ was computed from a trilinear interpolation of velocities at eight neighboring points. It should be noted that the estimated velocity gradient, or the shear stress, based on the above quantities is expected to be smaller than the actual value, since the distance $\Delta h$ is still larger than the thickness of the boundary layer of the flow.

\subsection{Experimental procedure}

Porcine eyes, from which the muscles around the eyeball had been eliminated, were mounted on Styrofoam blocks using metal pins. The Styrofoam blocks with eyeball were then submerged in a Plexiglas water container, as shown in Fig. 2, and filled with saline water. After submersion, an incision parallel to the iris plane was made at the peripheral part of the cornea by using a surgical knife, and the handpiece was inserted into the anterior chamber through the incision. Irrigation and aspiration were initiated, and particle images were recorded. Each of the two cameras captured 200 double images at a frequency of $30 \mathrm{~Hz}$. After the recording of images, the water container with the eyeball and the handpiece was displaced $0.5 \mathrm{~mm}$ in the horizontal direction by using a traversing 
mechanism, labeled as the $x-z$ stage in Fig. 2. This sequence of capturing the particle image and displacing the eyeball was repeated 27 times. After we captured 27 measurement planes, the irrigation fluid was replaced with saline water containing Rhodamine6G, so we could obtain the fluorescent images that we used to identify the position of the corneal surface. The fluorescent images were recorded at the 27 measurement planes whose locations were identical to those of the particle images.

\subsection{Error estimates}

The typical random or bias error associated with the computation of particle displacement is 0.1 pixels. This leads to an error in both the instantaneous and mean velocity of $12 \mathrm{~mm} / \mathrm{s}$. The error involved in the calculation of the mean velocity, which is the ensemble average over $N=200$ instantaneous velocity data, is estimated to be $N^{1 / 2} U_{\mathrm{rms}}=7 \mathrm{~mm} / \mathrm{s}$, where the value of the RMS velocity was assumed to be $U_{\mathrm{rms}}=100 \mathrm{~mm} / \mathrm{s}$, as shown in the following section.

Another source of error is associated with the spatial resolution, i.e., the size of the interrogation volume. In PIV the velocity is computed as an average of the particle velocity within the interrogation volume. Therefore, the velocity distribution of an eddy smaller than, or even comparable to, the interrogation volume size might be indicated as smoother and more damped in its amplitude. In the present study the interrogation volume size represented by the thickness of the light sheet, $1 \mathrm{~mm}$, was comparable to the width of the irrigation jet issued from the side port of the sleeve. Thus the measured velocity profile of the irrigation jet is expected to be significantly reduced from the true value. Our estimation indicated that the maximum error is approximately $50 \%$ of the true value when the velocity profile of the jet was assumed to be Gaussian and its half width was $0.25 \mathrm{~mm}$. This is a reasonable value for the error in the vicinity of the side port, and the error should be reduced downstream where the jet spreads out.

\section{Results}

We first present the results of the standard coaxial phacoemulsification. Figure 9 shows the mean velocity vectors for case SCA1. Figure 9(a) shows a bird's eye view of the velocity vectors in the $x$-z plane with streamlines. Although we have not measured the exact location of the surface of the sclera (white spherical part), a virtual model of the sclera was also overlaid on this figure for easier recognition of the whole geometry. After irrigation fluid was ejected from the side ports of the sleeve, fluid travels through the anterior chamber and then impinges on the edge of the anterior chamber. The angle between the flow directions of the two jets was approximately 60 degrees. The velocity component of $w$ in the $x-y$ plane at $z=2.5 \mathrm{~mm}$ shown in Fig. 9(b) exhibited two isolated peaks located approximately $5 \mathrm{~mm}$ apart, representing the irrigation jet issued from the sleeve. Here the velocity magnitude reaches approximately $210 \mathrm{~mm} / \mathrm{s}$ at $(x, y)=(1.8 \mathrm{~mm}, 1.5 \mathrm{~mm})$.

Note that the measured velocity immediately downstream of the side port was approximately $110 \mathrm{~mm} / \mathrm{s}$, which is a much lower value than that expected from the flow rate in the immediate area of the side port, due to the lack of spatial resolution of the measurement, as discussed in Sec.2.7. Since the length scale of the jet increases as traveling downstream, the spatial 
resolution becomes more adequate for resolving the scale and therefore the value of the measured velocity increases. The measured velocity eventually reaches its maximum and then starts to decay by momentum diffusion. Table 2 summarizes the maximum value of the measured velocity along the core of the jet. This table also shows the cross-sectional area, which is represented by the area where the velocity magnitude is larger than half the maximum velocity, of the core of the jet when the measured velocity reaches maximum. The Reynolds number based on the equivalent radius of the cross-sectional area and the maximum measured velocity is also shown in Table 2 . The Reynolds number ranges from approximately 100 to 300 .

Fig. 9(c) shows the same velocity vectors as are shown in Fig. 9(b), except in this case the vectors are overlaid with color contour lines showing the level of fluctuation represented by the root-mean square value of the $w$-component of instantaneous velocity vectors. The RMS value also shows two distinct peaks at the location where the mean velocity reaches local maximums. The peak RMS value is approximately $100 \mathrm{~mm} / \mathrm{s}$, which is about half the local mean velocity. The velocity fluctuation was expected, due to the unsteady behavior of the jet column. According to Crow and Champaign [13], the round jet close to the nozzle exit in a range of $\mathrm{Re}=100-1000$, which covers the Reynolds number of the present case, exhibits undulation of the laminar column of the jet rather than rapid development into a fully turbulent regime. Note that the unsteady motion or vibration of the cornea surface was not visible during the experiments. The standard deviation of the instantaneous location of the cornea surface identified from the LIF image was less than 0.1 $\mathrm{mm}$.

The velocity distribution in the $y-z$ plane shown in Fig. 9(d) depicts the flow along the corneal surface. Fluid issued from the handpiece immediately flows along the cornea and then moves back toward the handpiece with the retardation of velocity. The viscous shear stress acting on the interior surface of the cornea projected on the $x$-z plane is presented in Fig. 9(e). It clearly shows two distinct peaks of the shear stress at $(x, y)=(-2.5 \mathrm{~mm}, 5 \mathrm{~mm})$ and $(2 \mathrm{~mm}, 4 \mathrm{~mm})$, where the irrigation fluid first impinges on a surface right after its ejection from the sleeve. The maximum value of the shear stress reached $0.28 \mathrm{~Pa}$, as listed in Table 2 along with that of other cases.

The flow under case SCA2 shown in Fig. 10(a) represents a flow structure similar to that of case SCA1, but it is slightly asymmetric with respect to the $y$-z plane. The asymmetric flow pattern is obvious in the vector plot in the $x-y$ plane at $z=3.5 \mathrm{~mm}$, as shown in Fig. 10(b). The right- and left-hand-side irrigations in the figure can be observed as peaks of the $w$-component of velocity at $(x, y)=(-3 \mathrm{~mm},-1 \mathrm{~mm})$ and $(x, y)=(3 \mathrm{~mm}, 0.5 \mathrm{~mm})$, respectively. Both irrigations are asymmetric, and the right-side irrigation velocity in the figure is meaningfully higher than the leftside irrigation velocity. Although both irrigation ports made on the sleeve have the same dimensions, there was an uneven flow rate through the port. This might have been due to nonuniform gap spacing between the sleeve and the tip, since the sleeve could easily have been deformed when it was inserted through the incision. The shear stress distribution was also asymmetric with respect to the z-axis, as shown in Fig. 10(c). The viscous shear stress exhibited its highest value, $0.21 \mathrm{~Pa}$, in the region where the irrigation flow impinged upon the cornea. 
While the above cases have direct impingement of the irrigation fluid on the cornea, case SCA3, in which the head of the handpiece went deeper than in other cases, exhibits different features of flow pattern. Figure 11(a) shows that the irrigation fluid impinged up to the edge of the cornea and then returned through the middle of two irrigation jet flows toward the head of the handpiece along the cornea. The return flow with lower velocity along the cornea resulted in lower viscous shear stress on the cornea, as shown in Fig. 11(b). The shear stress was more uniformly distributed than in the other cases, although a higher value can be recognized in a small area near the edge.

Flow fields created by micro-coaxial phacoemulsification are presented in Figs. 12-14. The velocity field under case MCA1 shown in Fig. 12(a) depicts a significantly asymmetric flow pattern formed by a circulatory flow along the edge of the cornea. Although the sleeve has two side ports similar to the standard coaxial type, the uneven flow rate through the port is more significant than that in case SCA2 shown previously. An asymmetric flow pattern is also evident in case MCA2 in Fig. 13(a). While cases MCA1 and MCA2 had relatively higher values of shear stress in regions directly impinged on by irrigation, case MCA3 showed a lower value of shear stress, $0.18 \mathrm{~Pa}$ at maximum, as shown in Fig. 14(b). In this case the irrigation flow impinged on a deeper region below the cornea, avoiding direct impingement on the cornea surface, since the handpiece was placed farther away from the cornea than in cases MCA1 and MCA2. After the impingement, the flow returned towards the tip of the handpiece through the middle of the posterior chamber along the interior surface of the cornea, where the maximum shear stress was observed. Such a flow pattern is much like the observation of case SCA3 shown in Fig. 11.

\section{Discussion}

Previous studies have qualitatively and quantitatively evaluated the fluid flow induced by ultrasound tips during phacoemulsification. Tognetto et al. [14] used a Fourier optics-based phase image to visualize the qualitative flow pattern created near the tip. When irrigation was activated in the absence of aspiration/sonication, the laminar flow pattern originated from the irrigation port of the phacoemulsification sleeve. Steinert et al. [5] used the ultrasonic Doppler method to measure the planar velocity distribution of the irrigation flow near the phacoemulsification tip. The results indicated there was fluid movement, which was produced by a nonlinear effect known as acoustic streaming, directly below the phacoemulsification tip. Oki [15] used the schlieren method to visualize the flow near the tip and found that sonification caused rectilinear flow patterns.

While the above studies clarified the detailed flow features near the phacoemulsification tip, studies of the actual flow field that occurs in the eye have not yet been published. To the best of our knowledge, the results of the present study document for the first time the quantitative velocity distribution in the anterior chamber during irrigation and aspiration. The time-averaged velocity field that was reconstructed from the multiple cross-sectional data represents three-dimensional flow behaviors in the eye. 
Collecting quantitative velocity data enabled us to evaluate the viscous shear stress applied on the corneal endothelial surface. Kaji et al. [10] found there was an increased detachment of the corneal endothelial cells when the shear stress was above $0.1 \mathrm{~Pa}$, which is comparable to the maximum shear stress $(0.45 \mathrm{~Pa})$ we observed in the present study. In addition, shear stress has also been shown to affect other endothelial cells such as vascular endothelial cells [8] and hybridomas [9].

It should be noted that the actual shear stress could be larger than the estimated values. Since the estimated values for the shear stress were computed from the velocity gradient in the vicinity of the corneal surfaces, any error involving the velocity gradient would directly affect the shear stress value. The spatial resolution of the present measurement was approximately $1 \mathrm{~mm}$ in the $\mathrm{z}$ direction, while the thickness of the boundary layer that developed on the corneal surface was estimated to be of the same order of magnitude. Therefore, knowing the spatial resolution is not sufficient for estimating the velocity gradient computed via the numerical differentiation of the measured velocity field. Lack of spatial resolution causes a spatial filtering effect on the measured velocity field, which results in underestimation of the velocity gradient and, consequently, the shear stress.

The hydrodynamics of the irrigation fluid during real cataract surgeries are far more complicated than those of our current model. Since the motion of the tracer particle does not perfectly represent that of the fluid, we measured the velocity distribution without sonification. The frequency response of the tracer particle against the fluid was approximately $10^{5} \mathrm{~s}^{-1}$, which is inadequate when trying to follow the vibration of the fluid element in an ultrasonic frequency $\left(\sim 10^{7} \mathrm{~s}^{-1}\right)$. Note that the typical fluid velocity of the ultrasound-induced flow given in the article by Steinert et al. [5] is approximately $20 \mathrm{~mm} / \mathrm{s}$ when the ultrasound power was set at $70 \%$. This velocity is somewhat smaller than the irrigation velocity observed in the present results.

Although porcine eyes have been used by ophthalmologists for their practice operations for many years, since the dimensions are similar to those of human eyes, the discrepancy in the dimensions between the two types of eye should be addressed. The typical diameters of the internal anterior chambers of human and porcine eyes are $12.5 \mathrm{~mm}$ [16] and $13.6 \mathrm{~mm}$ [17], respectively. While the difference of the diameter is within $10 \%$, the depth of the anterior chamber of the porcine eye is $2.13 \mathrm{~mm}$ [17], which is $27 \%$ smaller than that of the human eye, $2.91 \mathrm{~mm}$ [18]. Such a discrepancy in the geometry may cause different values of the velocity and shear stress between porcine and human eyes. Since the shear stress is inverse proportional to the length scale of the flow, the eye model having smaller depth of the anterior chamber, i.e., the porcine eye, might result in a larger shear rate than that of the human eye. Note that the diameter of the pupil of an enucleated porcine eye is in the middle of the maximum and minimum values for the live pig. During phacoemulsification, however, the pupil becomes dilated, and its diameter becomes similar to that of the human eye. 
Although the tip of the handpiece was fixed in a position in the present study, it is possible that the flow pattern could be sensitive to the position and orientation of the tip. In addition, removal of the crystalline lens can alter the flow pattern due to a change in the shape of the posterior chamber. The absence of ophthalmic viscosurgical devices (OVD) in the current study also could have affected our results. The use of OVD would probably attenuate the turbulence intensity, which is known as Tom's effect, and consequently reduce the turbulent shear stress.

In conjunction with the above issues, evaluation of the hydrodynamic shear stress based on the measured velocity data is important when attempting to clarify the mechanics of the corneal damage that occurs during phacoemulsification.

\section{Conclusion}

Velocity distributions in the anterior chamber of porcine eyes under simulated cataract surgery with standard coaxial phacoemulsification and micro coaxial phacoemulsification were measured using stereo-PIV. An asymmetric flow rate of irrigation was observed with both procedures, although both irrigation ports had the same dimensions prior to insertion of handpieces into the eye. An especially strong asymmetric irrigation flow rate was observed with simulated micro coaxial phacoemulsification. In cases where the tip of the handpiece was placed farther from the cornea, i.e., closer to the crystalline lens, direct impingement of irrigation flow on the cornea surface was avoided and the flow returned towards the handpiece along the cornea surface. The viscous shear stress on the corneal endothelium was computed based on the measured mean velocity distribution. The magnitude of shear stress for micro coaxial cases with direct impingement of the irrigation flow on the cornea reached $0.45 \mathrm{~Pa}$, which is comparable to the value of shear stress that caused detachment of the corneal endothelial cells reported by Kaji et al.[10]. By avoiding direct impingement of the irrigation flow, the shear stress was reduced considerably.

\section{Acknowledgement}

The support of the Japan Society for the Promotion of Science under Grant No.19360082 is gratefully acknowledged.

\section{References}

1. Hayashi K, Hayashi H, Nakao F, Hayashi F. Risk factors for corneal endothelial injury during phacoemulsification. J Cataract Refract Surg 1996; 22: 1079-1084

2. O'Brien PD, Fitzpatrick P, Kilmartin DJ, Beatty S. Risk factors for endothelial cell loss after phacoemulsification surgery by a junior resident, J Cataract Refract Surg 2004; 30: 839-843

3. Topaz M, Motiei M, Assia E. Acoustic cavitation in phacoemulsification: chemical effects, modes of action, and cavitation index. Ultrasound Med Biol 2002; 28: 775-784

4. Miller MW, Miller DL, Brayman AA. A review of in vitro bioeffects of inertial ultrasonic cavitation from a mechanistic perspective. Ultrasound Med Biol 1996; 22: 1131-1154 
5. Steinert RF, Schafer ME. Ultrasonic-generated fluid velocity with Sovereign WhiteStar micropulse and continuous phacoemulsification. J Cataract Refract Surg 2006; 32: 284-287

6. Polack FM, Sugar A. The phacoemulsification procedure. II. Corneal endothelial changes. Invest Opthalmol 1976; 15: 458-469

7. Binder PS, Sternberg H, Wickham MG, Worthen DM. Corneal endothelial damage associated with phacoemulsification. Am J Ophthalmol 1976; 82: 48-54

8. Thoumine O, Znegler T, Girard PR, Nerem RM. Elongation of confluent endothelial cells in culture: the importance of fields of force in the associated alterations of their cytoskeletal structure. Exp Cell Res 1995; 219: 427-441

9. Born C, Zhang Z, Al-Rubeai M, Thomas CR. Estimation of disruption of animal cells by laminar shear stress. Biotechnol Bioeng 1992; 40: 1004-1010

10. Kaji Y, Oshika T, Usui T and Sakakibara J. Effect of shear stress on attachment of corneal endothelial cell in association with corneal endothelial cell loss after laser iridotomy. Cornea 2005; 24: S55-S58

11. Adrian RJ. Twenty years of particle image velocimetry, Exp Fluids 2005; 39: 159-169

12. Sakakibara J, Nakagawa M, Yoshida M. Stereo-PIV study of flow around a maneuvering fish. Exp Fluids 2004; 36: 282-293

13. Crow, S.C., Champagne, F.H. Orderly structure in jet turbulence. J Fluid Mech 1971; 48:547591

14. Tognetto D, Sanguinetti G, Sirotti P, Brezar E, Ravalico G. Visualization of fluid turbulence and acoustic cavitation during phacoemulsification. J Cataract Refract Surg 2005; 31: 406411

15. Oki K. Measuring rectilinear flow within the anterior chamber in phacoemulsification procedures. J Cataract Refract Surg 2004; 30: 1759-1767

16. Kohnen T, Thomala MC, Cichocki M, Strenger A. Internal anterior chamber diameter using optical coherence tomography compared with white-to-white distances using automated measurements. J Cataract Refract Surg 2006; 32:1809-1813

17. Asejczyk-Widlicka M., Schachar RA, Pierscionek BK. Optical coherence tomography measurements of the fresh porcine eye and response of the outer coats of the eye to volume increase. J. Biomed Opt 2002; 13: 024002

18. Saw SM, Wong TY, Ting S, Foong AWP, Foster PJ. The relationship between anterior chamber depth and the presence of diabetes in the Tanjong Pagar Survey. Am $J$ Ophthalmology 2007; 144:325-326 


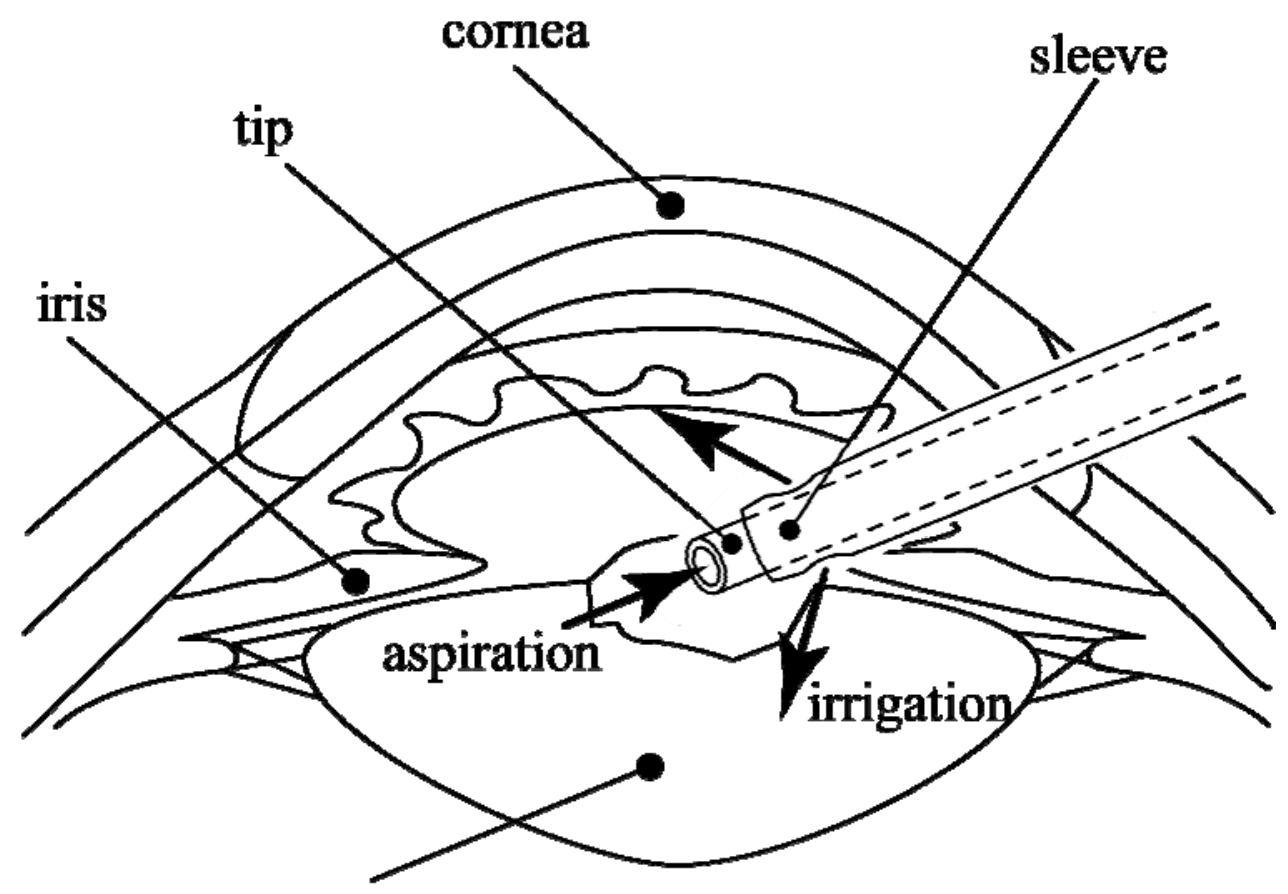

crystalline lens

Figure 1 Schematic of phacoemulsification cataract surgery. 

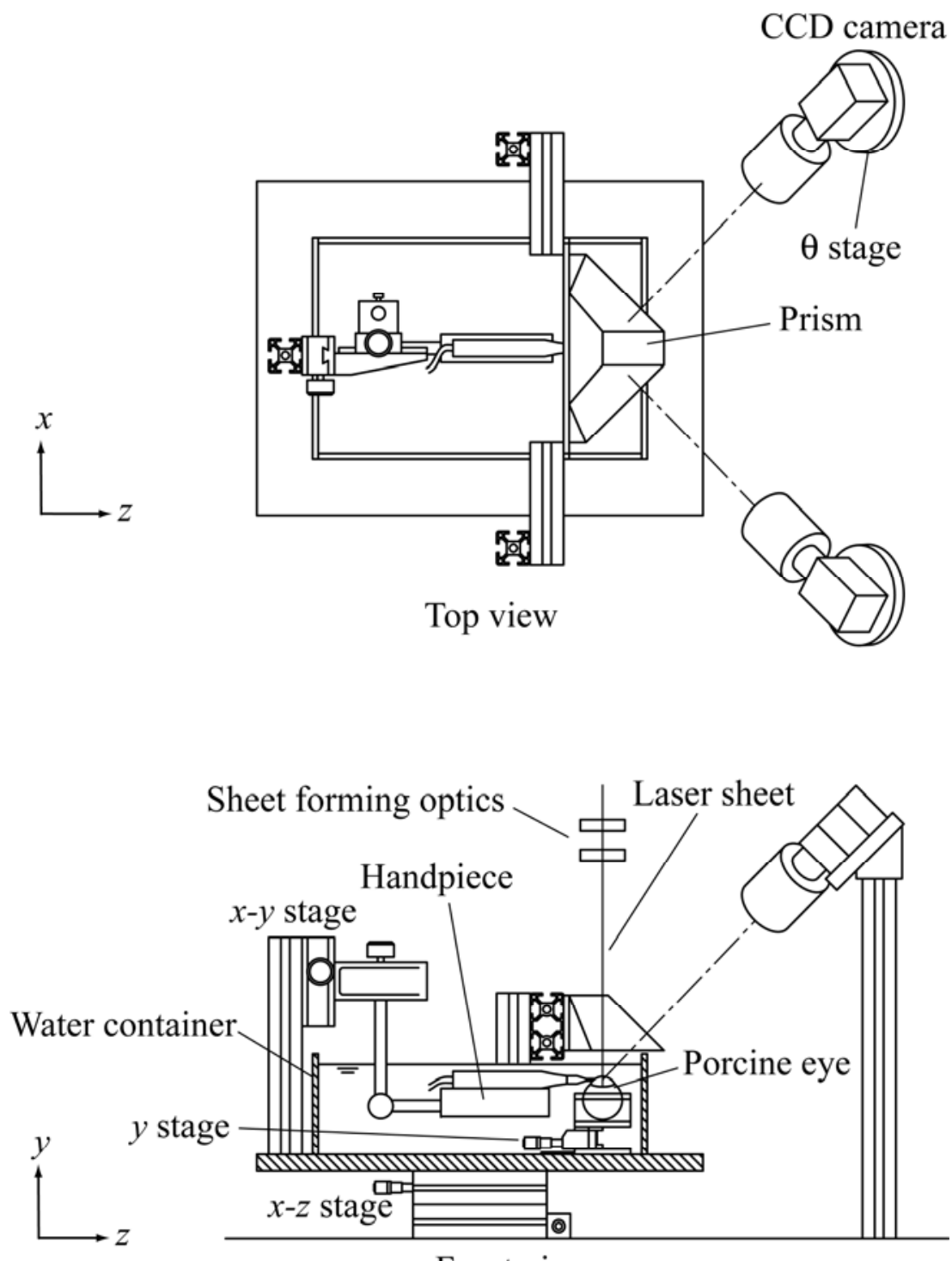

Front view

Figure 2 Overall view of the experiment. 


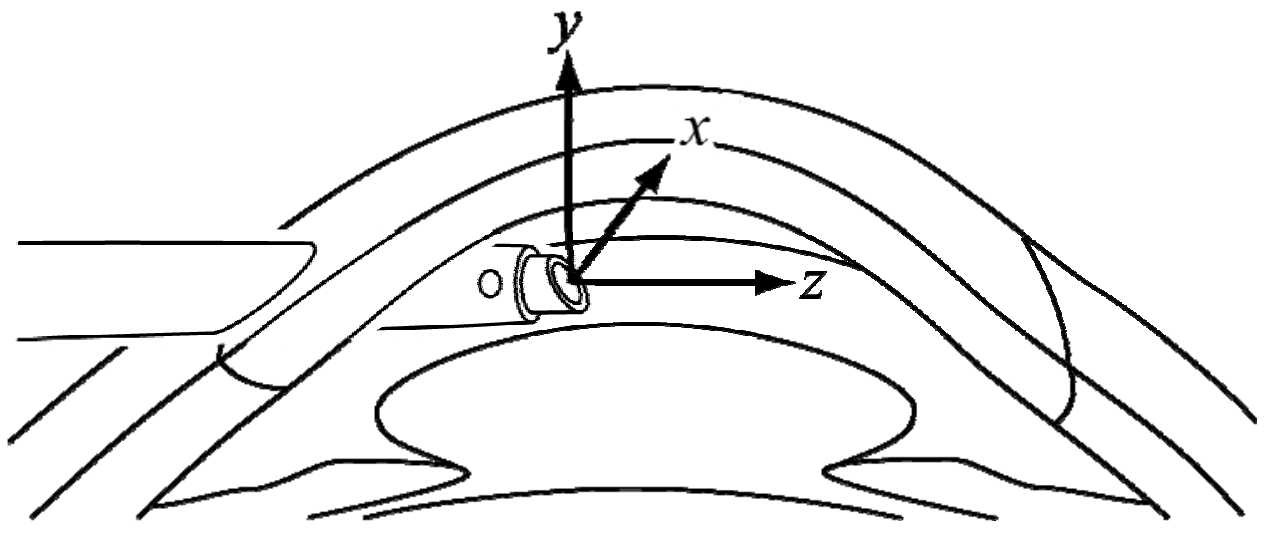

Figure 3 Definition of the coordinate system. 

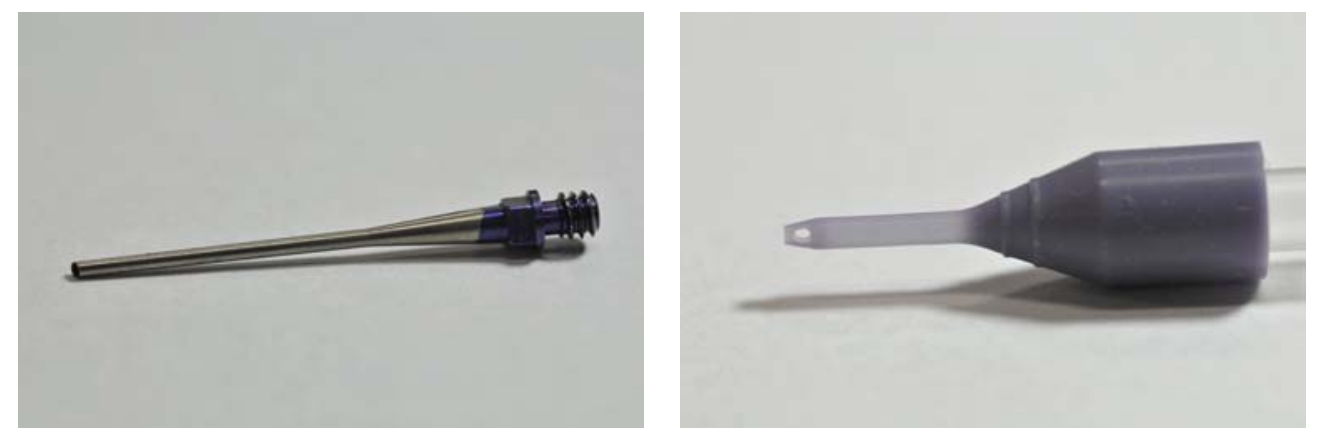

(a) $0.9 \mathrm{~mm}$ micro ABS tip $30^{\circ}$

(b) $0.9 \mathrm{~mm}$ micro sleeve
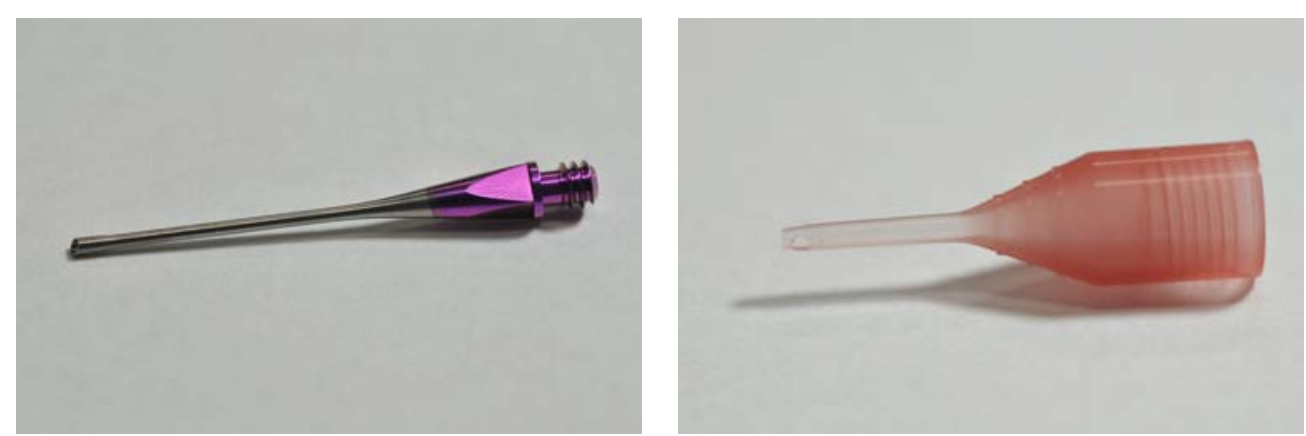

(c) $0.9 \mathrm{~mm}$ micro flared ABS tip $30^{\circ}$

(d) $0.9 \mathrm{~mm}$ ultra sleeve

Figure 4 Tips and sleeves. 
Table 1 Case names and descriptions

\begin{tabular}{|c|c|c|c|c|c|}
\hline \multirow[b]{2}{*}{$\begin{array}{l}\text { Case } \\
\text { name }\end{array}$} & \multirow[b]{2}{*}{$\begin{array}{l}\text { Type of } \\
\text { surgery }\end{array}$} & \multicolumn{4}{|c|}{ Location of the head of the tip } \\
\hline & & $\begin{array}{c}\text { Distance } \\
\text { from top } \\
\text { surface } \\
\text { of } \\
\text { cornea } \\
\text { [mm] }\end{array}$ & $\mathrm{a}[\mathrm{mm}]$ & $\mathrm{b}[\mathrm{mm}]$ & $\mathrm{c}[\mathrm{mm}]$ \\
\hline SCA1 & $\begin{array}{c}\text { standard } \\
\text { coaxial }\end{array}$ & 2.0 & 8.5 & 6.5 & 5.0 \\
\hline SCA2 & $\begin{array}{c}\text { standard } \\
\text { coaxial }\end{array}$ & 2.0 & 7.0 & 5.5 & 6.5 \\
\hline SCA3 & $\begin{array}{c}\text { standard } \\
\text { coaxial }\end{array}$ & 3.5 & 8.5 & 5.3 & 7.0 \\
\hline MCA1 & $\begin{array}{c}\text { micro } \\
\text { coaxial }\end{array}$ & 2.0 & 10.0 & 6.3 & 5.8 \\
\hline MCA2 & $\begin{array}{l}\text { micro } \\
\text { coaxial }\end{array}$ & 3.0 & 8.5 & 5.3 & 7.3 \\
\hline MCA3 & $\begin{array}{c}\text { micro } \\
\text { coaxial }\end{array}$ & 3.8 & 10.0 & 4.8 & 6.5 \\
\hline
\end{tabular}




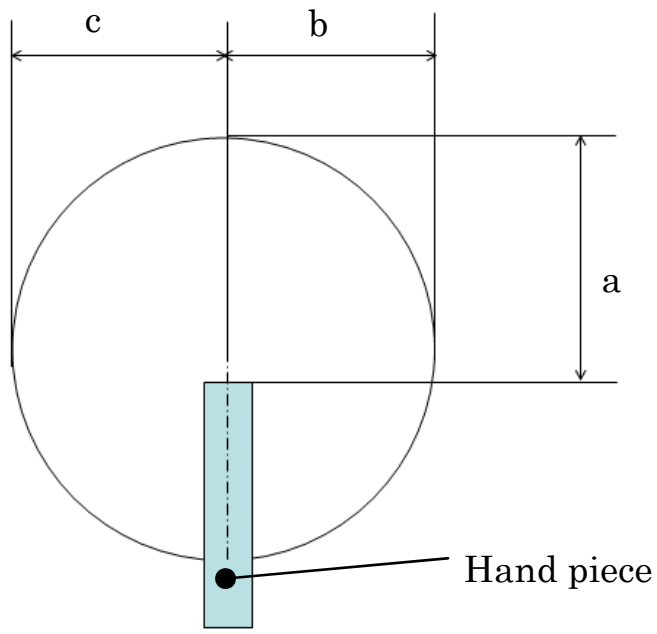

Figure 5 Parameters used to define the location of the head of the tip relative to the outer edge of the cornea. 


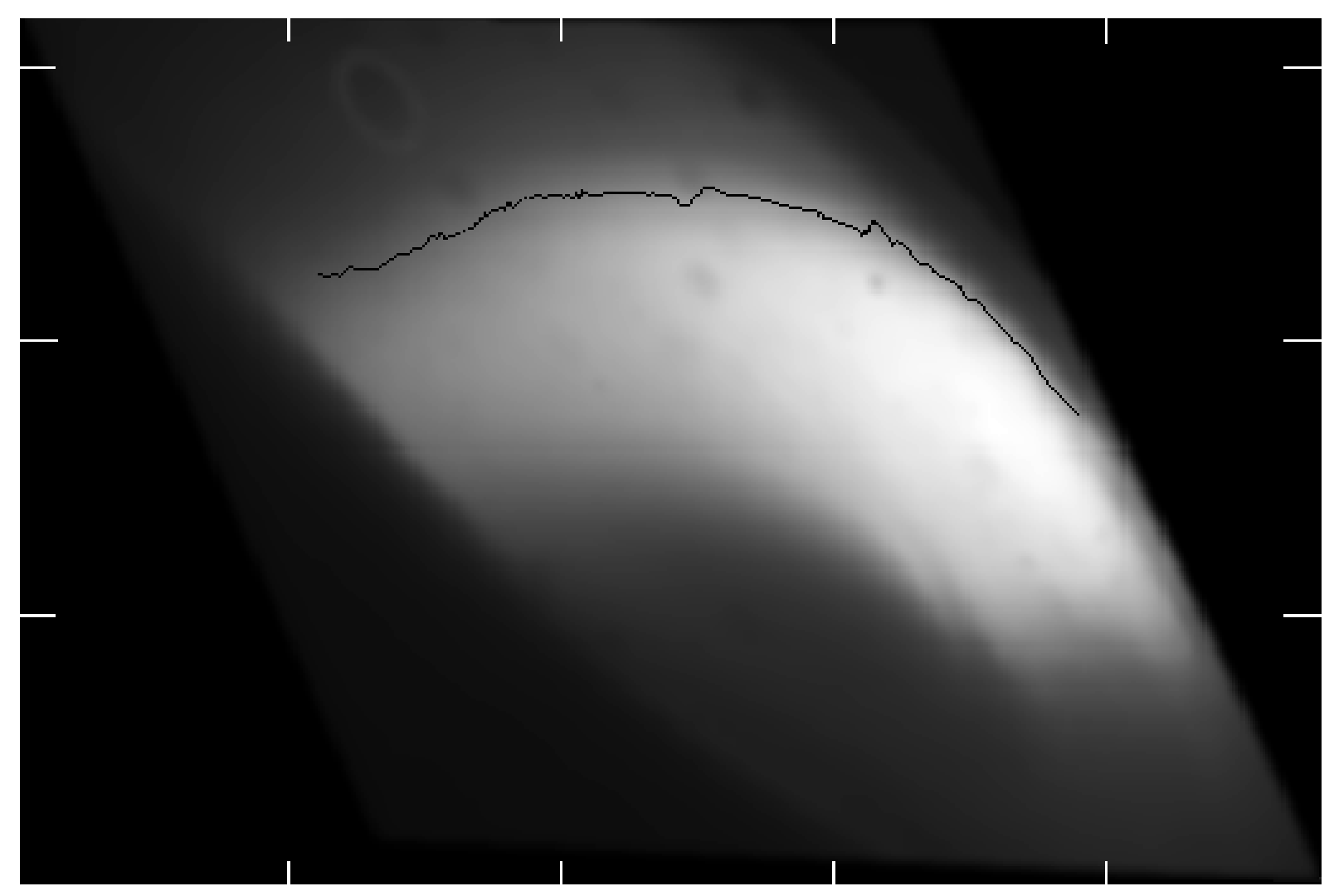

Figure 6 Ensemble-averaged fluorescence image used for determination of the location of the cornea surface. The original image was warped based on the calibration function to fit the physical coordinates. The solid line represents the locations of local inflection points. The tick marks are at 4-mm intervals. 


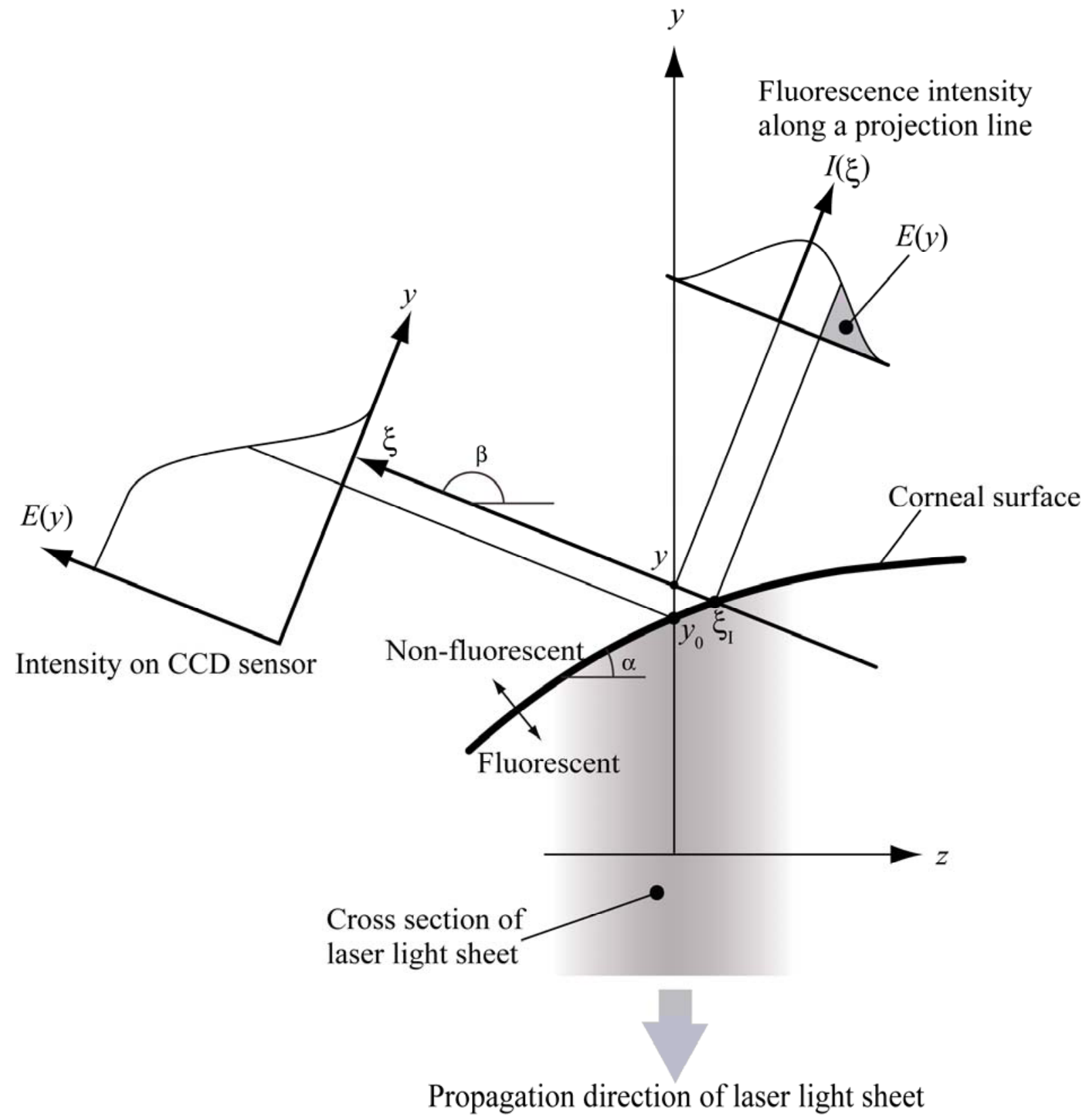

Figure 7 Schematic representation of the projection of the fluorescent light onto the camera. 


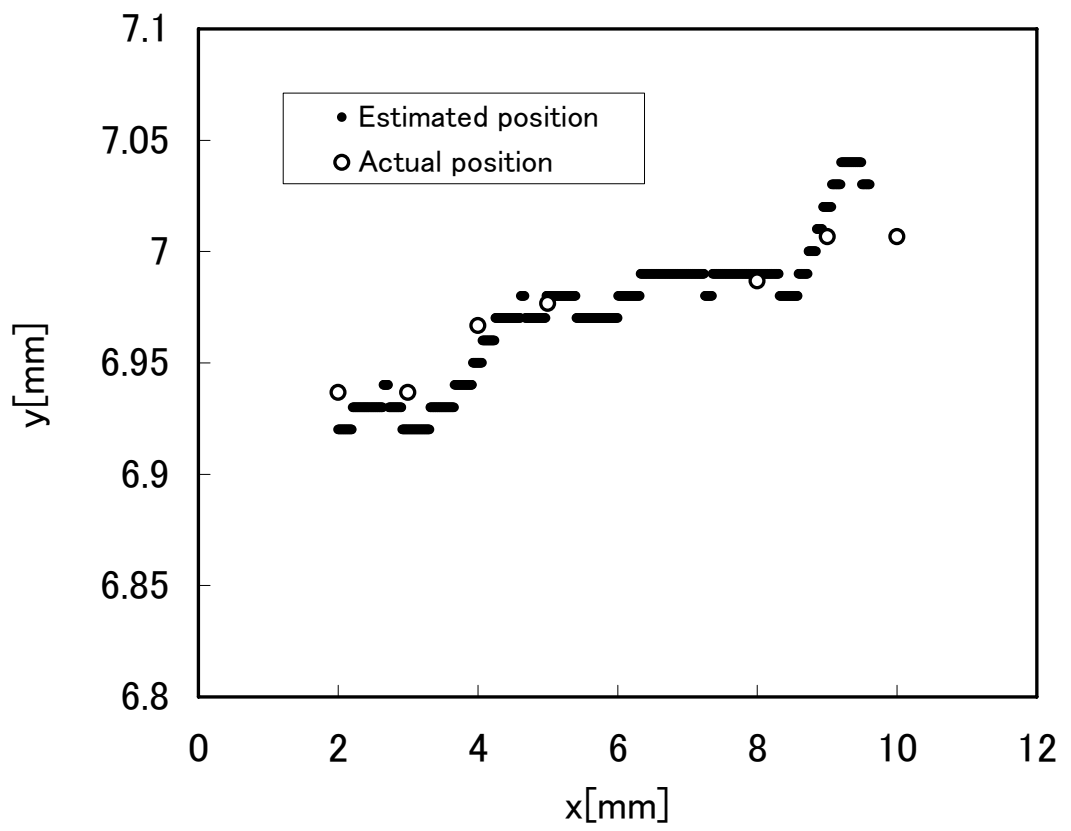

Figure 8 Location of the interior surface below the glass plate $(y)$ against the horizontal location $(x)$. 
(a)

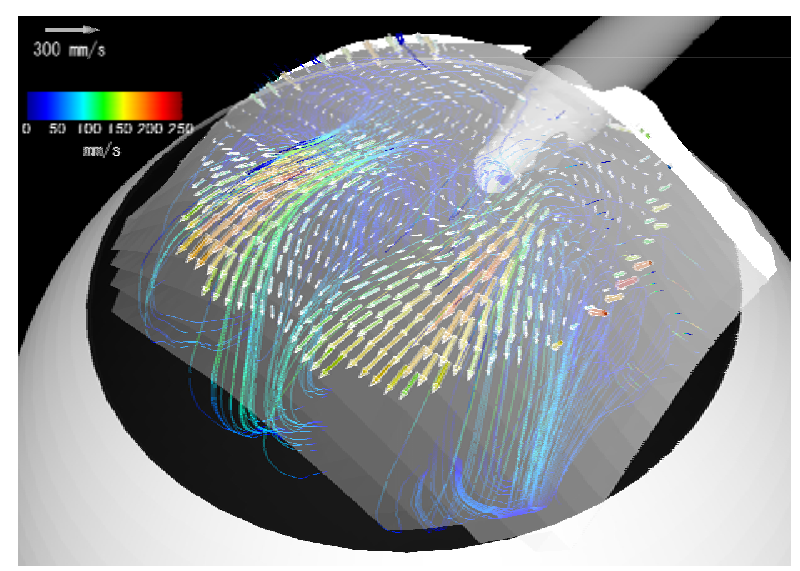

(c)

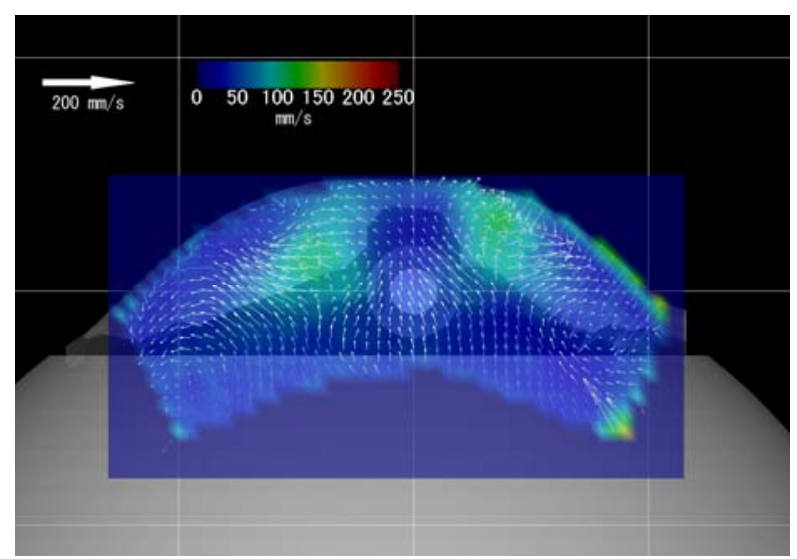

(e)

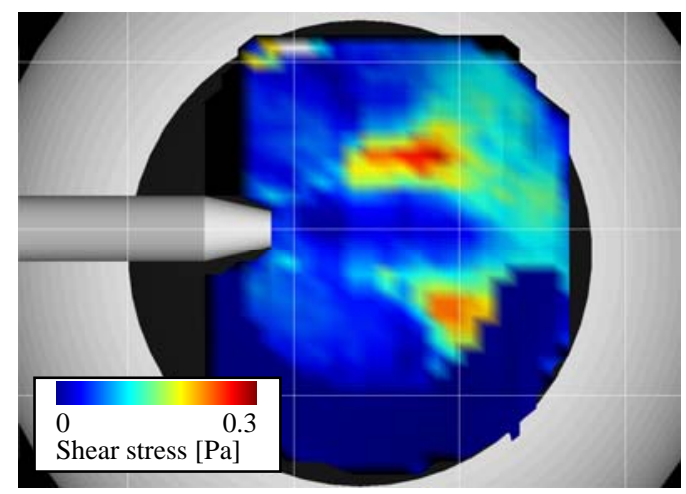

Fig. 9 Mean velocity vectors and other quantities for case SCA1. (a) Bird's-eye view of the mean velocity vectors $(y=0.75 \mathrm{~mm}$ ) with streamlines colored by the velocity magnitude, (b) mean velocity vectors in the $x$ - $y$ plane at $z=2.5 \mathrm{~mm}$ with the color scale representing the $w$-component of the mean velocity vectors, (c) mean velocity vectors in the $x-y$ plane at $z=2.5 \mathrm{~mm}$ with the color scale representing the RMS value of the $w$-component of velocity, (d) mean velocity vectors in the $y$-z plane at $x=0.25 \mathrm{~mm}$ with color representing the velocity magnitude, (e) viscous shear stress on the cornea surface projected on the $x$-z plane. The grid interval is

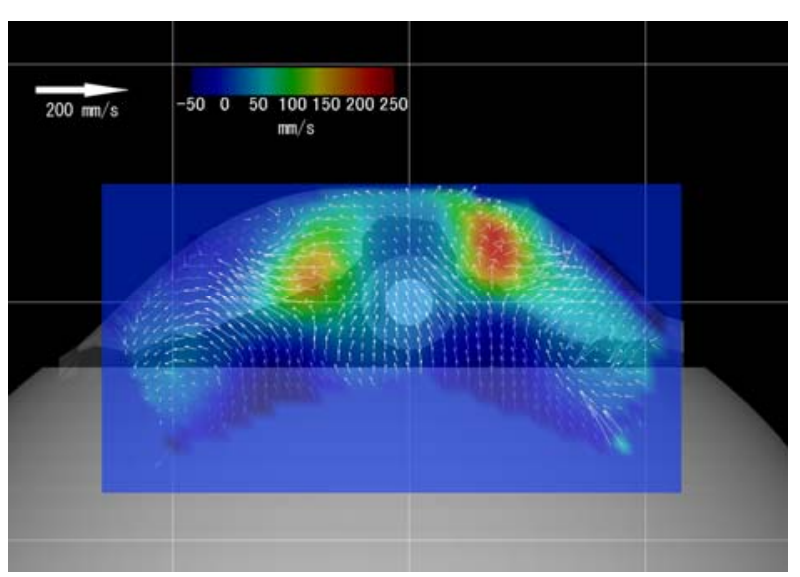

(d)

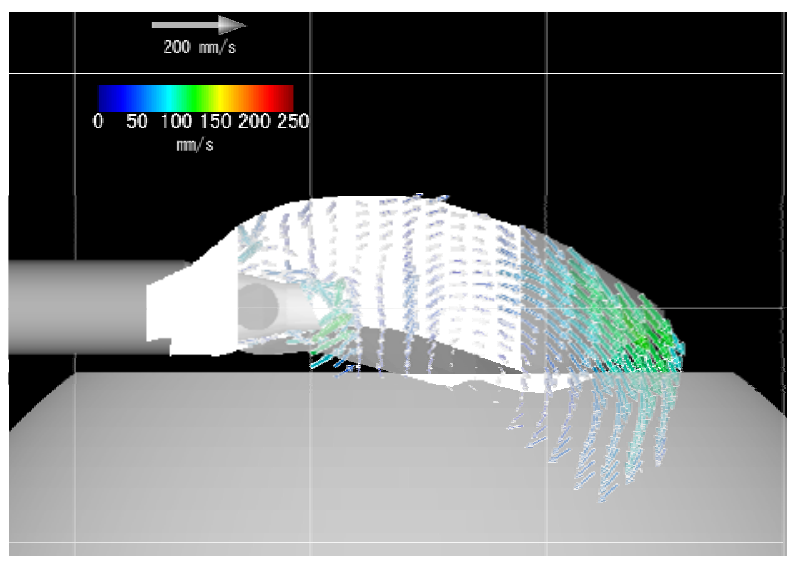


$5 \mathrm{~mm}$ in all directions. 
Table 2 Characteristics of the irrigation jet in conjunction with the maximum shear stress.

\begin{tabular}{lccccc}
\hline Case & $\begin{array}{c}\text { Maximum } \\
\text { velocity }[\mathrm{mm} / \mathrm{s}]\end{array}$ & $\begin{array}{c}\text { Velocity } \\
\text { half area } \\
{\left[\mathrm{mm}^{2}\right]}\end{array}$ & $\begin{array}{c}\text { Equivalent } \\
\text { radius } \\
{[\mathrm{mm}]}\end{array}$ & $R e$ & $\begin{array}{c}\text { Maximum viscous } \\
\text { shear stress [Pa] }\end{array}$ \\
\hline SCA1 & 233 & 40 & 0.89 & 208 & 0.28 \\
SCA2 & 260 & 43 & 0.92 & 241 & 0.21 \\
SCA3 & 210 & 12 & 0.49 & 102 & 0.086 \\
MCA1 & 440 & 28 & 0.75 & 328 & 0.45 \\
MCA2 & 309 & 48 & 0.98 & 302 & 0.34 \\
MCA3 & 287 & 29 & 0.76 & 218 & 0.18 \\
\hline
\end{tabular}


(a)

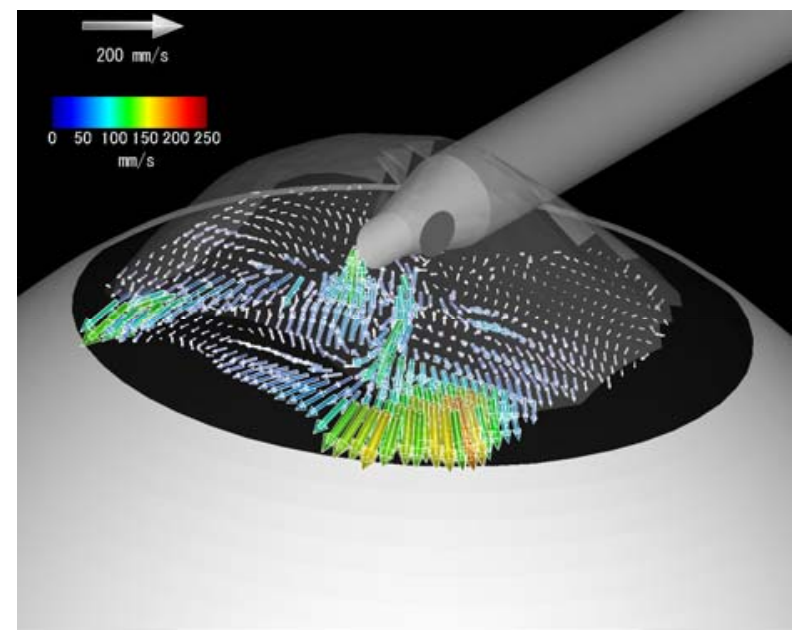

(b)

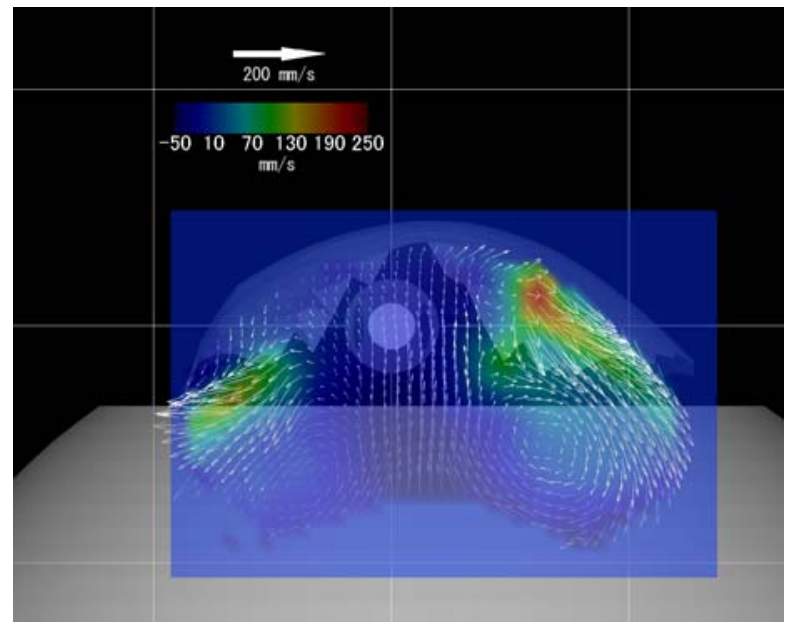

(c)

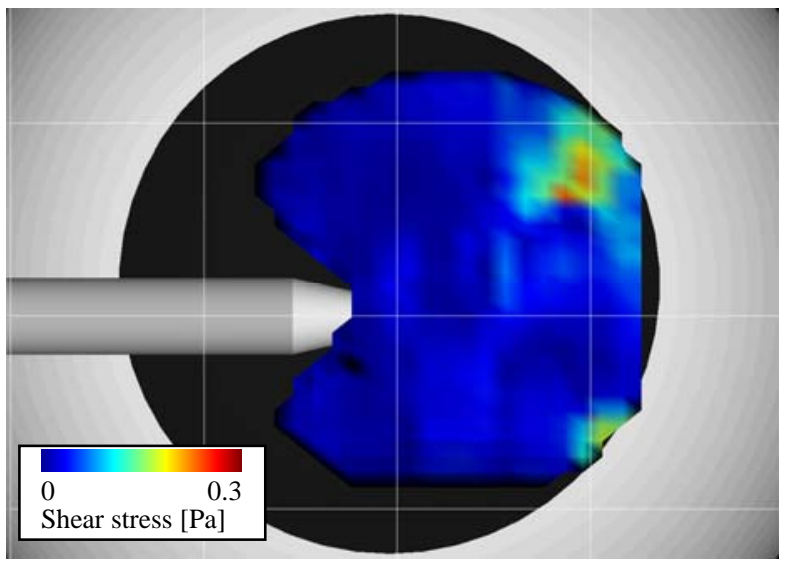

Fig. 10 Mean velocity vectors and other quantities for case SCA2. (a) Bird's-eye view of the mean velocity vectors $(y=0.75 \mathrm{~mm})$ colored by the velocity magnitude, (b) mean velocity vectors in the $x-y$ plane at $z=3.5$ mm with the color scale representing the w-component of the mean velocity vectors, (d) viscous shear stress on the cornea surface projected on the $x$-z plane. The grid interval is $5 \mathrm{~mm}$ in all directions. 
(a)

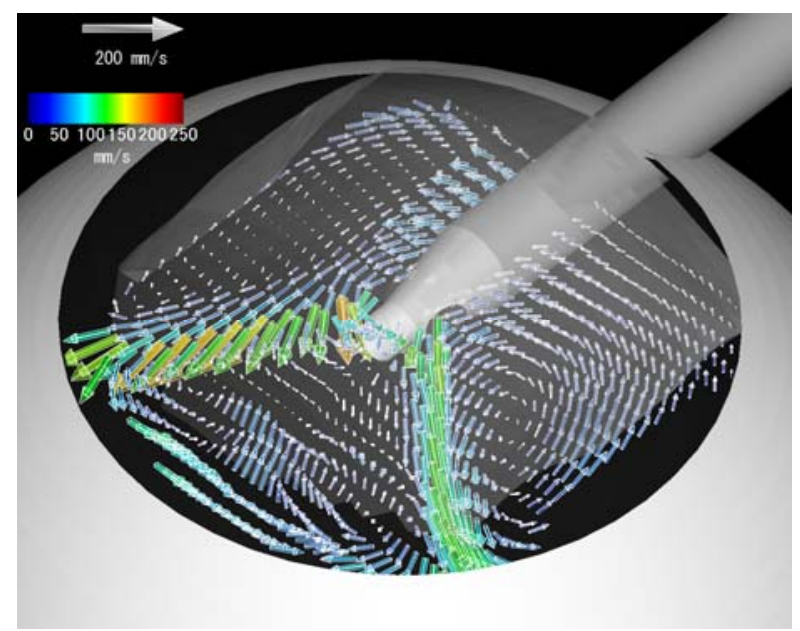

(b)

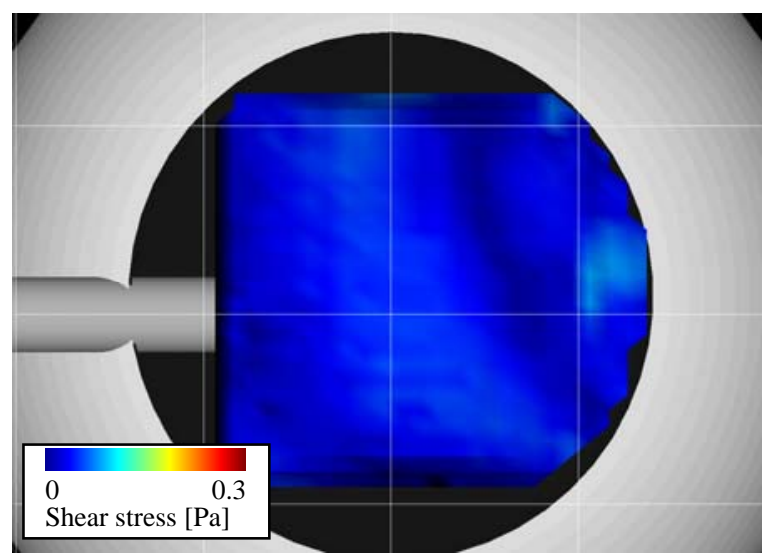

Fig. 11 Mean velocity vectors and shear stress for case SCA3. (a) Bird's-eye view of the mean velocity vectors $(y=0.25 \mathrm{~mm})$ colored by the velocity magnitude, (b) viscous shear stress on the cornea surface projected on the $x$-z plane. The grid interval is $5 \mathrm{~mm}$ in all directions. 
(a)

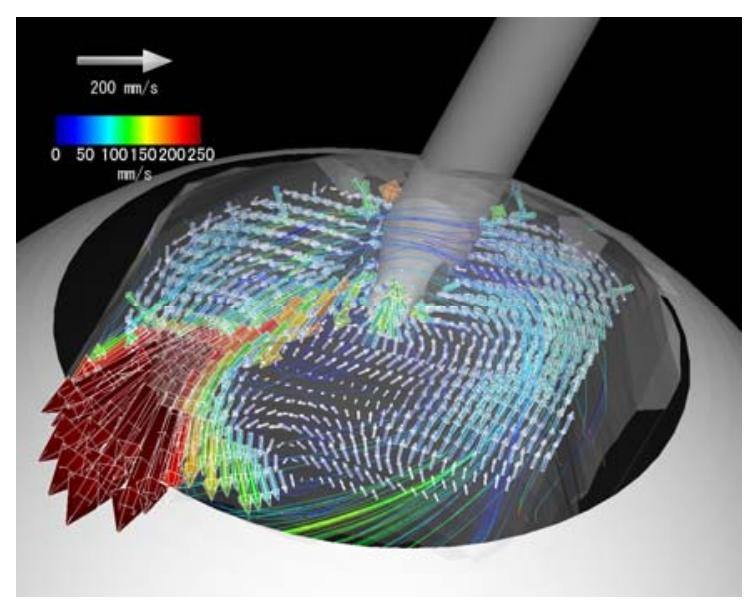

(b)

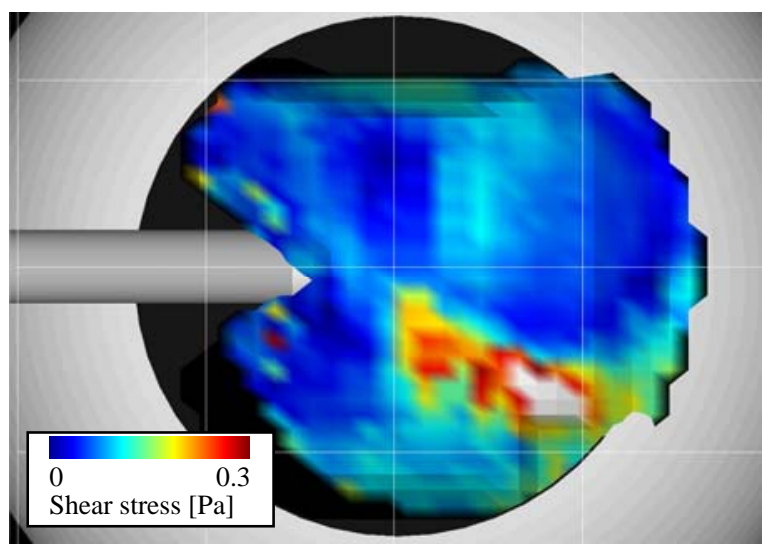

Fig. 12 Mean velocity vectors and shear stress for case MCA1. (a) Bird's-eye view of the mean velocity vectors $(y=0.5 \mathrm{~mm})$ and streamlines colored by the velocity magnitude, (b) viscous shear stress on the cornea surface projected on the $x$-z plane. The grid interval is $5 \mathrm{~mm}$ in all directions. 
(a)

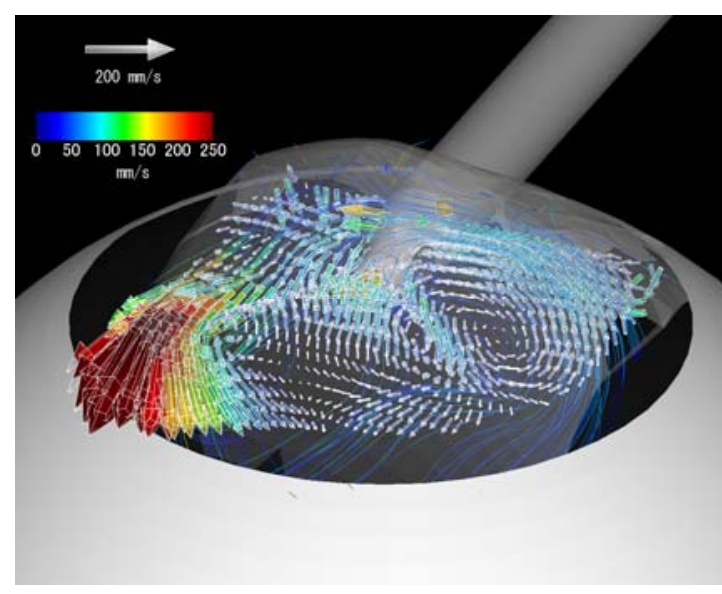

(b)

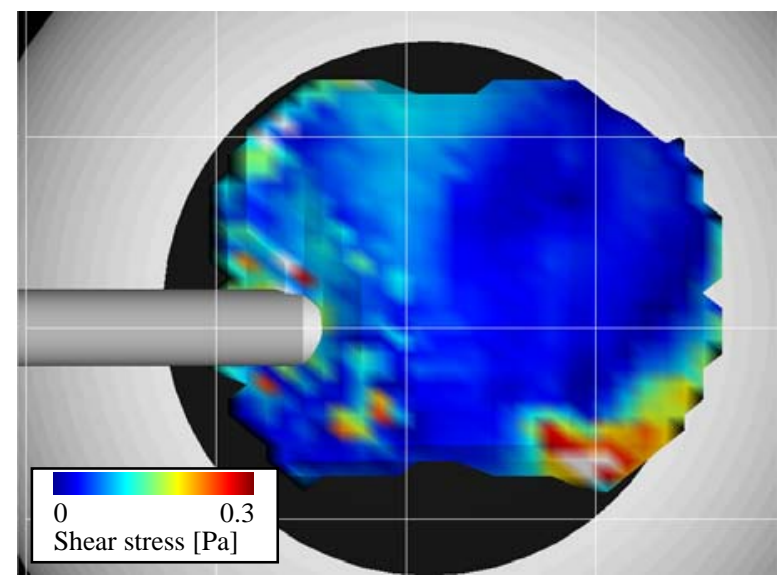

Fig. 13 Mean velocity vectors and shear stress for case MCA2. (a) Bird's-eye view of the mean velocity vectors ( $y=0.5 \mathrm{~mm}$ ) and streamlines colored by the velocity magnitude, (b) viscous shear stress on the cornea surface projected on the $x$-z plane. The grid interval is $5 \mathrm{~mm}$ in all directions. 
(a)

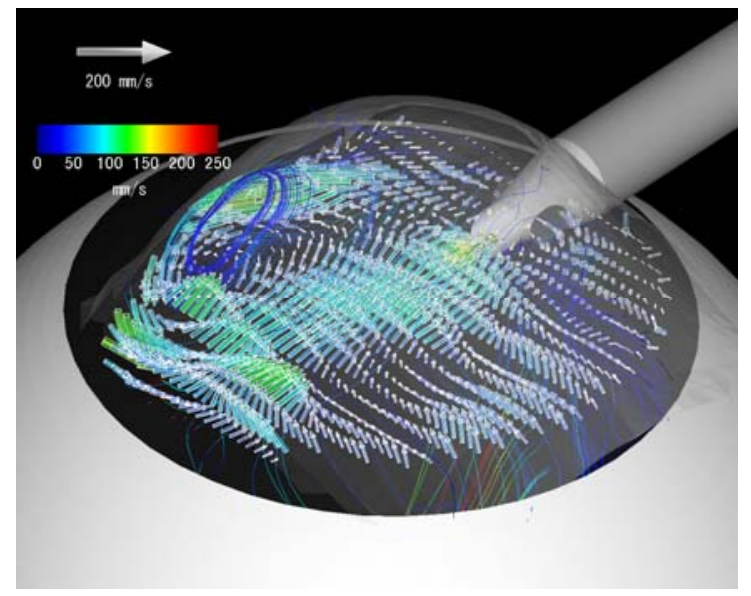

(b)

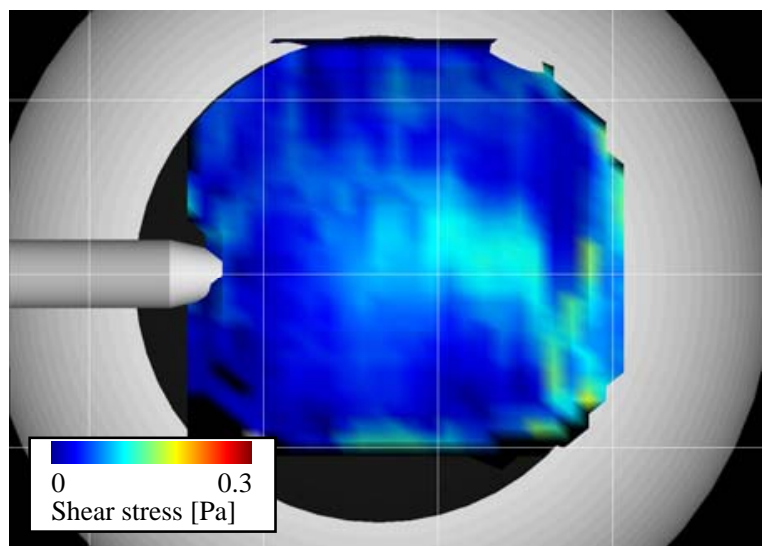

Fig. 14 Mean velocity vectors and shear stress for case MCA3. (a) Bird's-eye view of the mean velocity vectors ( $y=0 \mathrm{~mm}$ ) and streamlines colored by the velocity magnitude, (b) viscous shear stress on the cornea surface projected on the $x$-z plane. The grid interval is $5 \mathrm{~mm}$ in all directions. 